\title{
Giant dielectric constants at the approach to the insulator-metal transition
}

\author{
Harald F. Hess and Keith DeConde \\ Joseph Henry Laboratory, Princeton, New Jersey 08544 \\ T. F. Rosenbaum* and G. A. Thomas \\ Bell Laboratories, Murray Hill, New Jersey 07974
}

(Received 25 February 1982)

\begin{abstract}
We have measured the real and imaginary parts of the dielectric susceptibility of insulating samples of P-doped Si at millikelvin temperatures at $400 \mathrm{MHz}$ using a resonant transmission cavity. We find that the real part is enhanced by more than two orders of magnitude over the isolated donor polarizability, and we determine the exponent which describes the critical divergence of the real part at the insulator-metal transition by fitting the temperature dependence of the corresponding imaginary part. The form of the observed divergence remains unexplained theoretically.
\end{abstract}

The nonanalytic behavior of the zero-temperature conductivity in the metal and the divergence of the zero-temperature dielectric susceptibility in the insulator probe the nature of the metal-insulator transition in random systems. We report here complex susceptibility measurements at millikelvin temperatures on a series of bulk samples of Si:P which map out the divergence of the donor dielectric susceptibility as the critical density $n_{c}$ is approached. We measure dielectric constants over 700 which represent an enhancement of up to 150 over the isolated-donor polarizability ${ }^{1,2}$ and up to 20 over the largest previously reported $^{2}$ for $\mathrm{Si}: \mathrm{P}$.

Earlier studies have extracted the donor dielectric susceptibility from absorption measurements in the far infrared ${ }^{2,3}$ or from low-frequency capacitance measurements. ${ }^{4}$ In the far infrared, the absorption was measured ${ }^{2}$ over a broad band of frequencies and then integrated using the Kramers-Kronig relation to obtain the zero-frequency susceptibility. The method has two limitations near the transition: First, the nearly metallic reflectivity requires a large correction; second, absorption at frequencies below the measured range becomes important. In capacitance measurements ${ }^{4}$ the frequencies are typically low enough ( 1 to $100 \mathrm{kHz}$ ) to be considered zero frequency, but near the transition the resistive part of the impedance dominates unless the temperature is very low. By using intermediate frequencies near $400 \mathrm{MHz}$ and temperatures down to $20 \mathrm{mK}$, our apparatus allows susceptibility measurements closer to the transition than previous work.

We have determined the complex susceptibility from the shift of the resonant frequency and from the transmission loss, i.e., the quality factor $Q$ of a reentrant resonant transmission cavity (shown in the insert to Fig. 1). The sample partially filled the capacitive section of the cavity, so there was a gap capaci- tance in series with the sample capacitance and a stray capacitance in parallel with that series combination. To isolate the sample capacitance from the others and from the cavity inductance, four frequency measurements were made. These were done with an empty cavity, with an In sample of known geometry, with the Si:P sample in the high-temperature, highconductivity range (skin depth much smaller than sample dimensions), and finally, with the sample in the low-temperature insulating range, where the skin depth is much larger than the sample size. Using this procedure, the dielectric susceptibility was measured

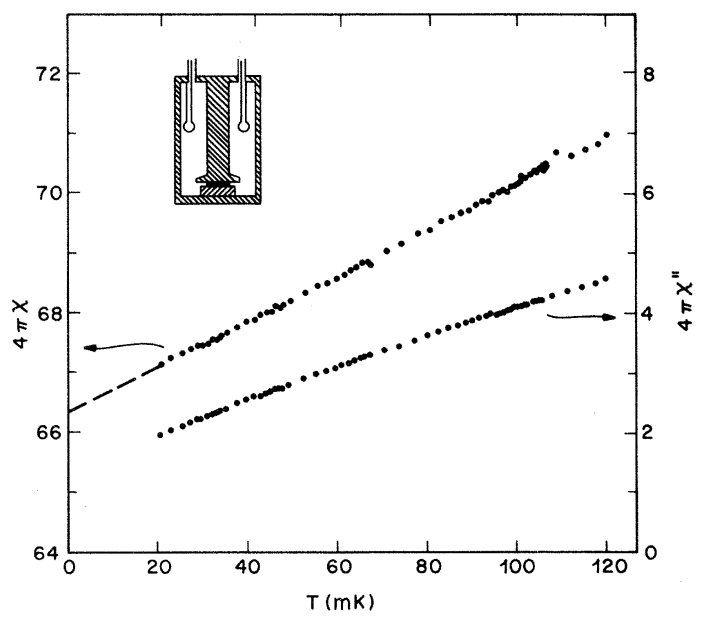

FIG. 1. Real and imaginary parts of the donor dielectric susceptibility as a function of temperature for $n=3.1 \times 10^{18}$ $\mathrm{cm}^{-3}$. The value of $4 \pi \chi(T)$ extrapolated to $T=0 \mathrm{~K}$ is shown in Fig. 2. The temperature dependence of $4 \pi \chi^{\prime \prime}$ is used to determine $T_{0}$ in Fig. 3. The inset shows the resonant cavity (cross hatched) with sample (black) and transmitting and receiving loops. 
with an absolute accuracy of better than $2 \%$. At all temperatures, the susceptibility was found to be independent of incident power for the nanowatt power levels used in these measurements. Figure 1 shows the temperature dependence of the donor dielectric susceptibility, $4 \pi \chi(T)=\epsilon-\epsilon_{\mathrm{Si}}=\epsilon-11.4$, of a Si:P sample with phosphorus donor density $n=3.1 \times 10^{18}$ $\mathrm{cm}^{-3}$. A linear extrapolation to $T=0 \mathrm{~K}$, as illustrated, determines $4 \pi \chi$. The figure also shows the temperature dependence of the imaginary part of the susceptibility $4 \pi \chi^{\prime \prime}(T)$, determined from the transmission loss of the cavity.

We confirm the suggestion of earlier work ${ }^{2,4}$ that $4 \pi \chi$ tends to diverge as $n$ approaches $n_{c}$ (Fig. 2). Our largest value of the dielectric susceptibility, 720, represents an enhancement by more than two orders of magnitude over the isolated-donor polarizability. $1,2,4$ The solid line is a fit to our data (solid circles) and that of Ref. 2 (open circles) of the form

$$
4 \pi \chi=\chi_{0}\left(n_{c} / n-1\right)^{-\zeta},
$$

with $\chi_{0}=7.0$, and $\zeta=1.15$. The value of $n_{c}=3.74 \times 10^{18} \mathrm{~cm}^{-3}$ is determined independently from measurements ${ }^{5}$ of the zero-temperature conductivity $\sigma(0)$ shown on the right side of Fig. 2. As $n$ approaches $n_{c}$ from above, $\sigma(0)$ approaches 0 . The

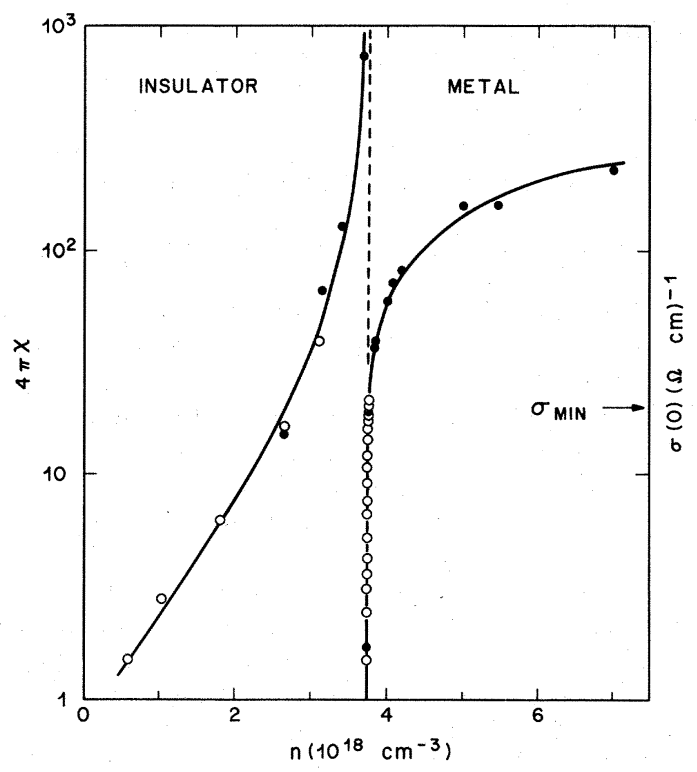

FIG. 2. Divergence of the $T=0 \mathrm{~K}$ donor dielectric susceptibility $4 \pi X$ in the insulator [solid circles, this work; open circles, Ref. 2; solid line, Eq. (1)] and the $T=0 \mathrm{~K}$ conductivity $\sigma(0)$ in the metal [solid circles, Refs. 5 and 6; open circles, Ref. 7; solid line, Eq. (2)] as a function of phosphorus donor density $n$. Together these results characterize the metal-insulator transition in a disordered system and indicate a tendency of $4 \pi \chi$ to diverge with a critical exponent assumed to be related to the divergence of the localization length. solid circles in Fig. 2 are obtained from millikelvin measurements 5,6 on samples of varying phosphorus concentration, while the open circles represent the response of one sample to uniaxial stress. ${ }^{7}$ The solid line through the $\sigma(0)$ is a fit of the form

$$
\sigma(0)=\sigma_{0}\left(n / n_{c}-1\right)^{\nu},
$$

with $\sigma_{0}=260(\Omega \mathrm{cm})^{-1}$ and $\nu=0.50$. Our choice of the forms of Eqs. (1) and (2) is motivated by the results of the scaling theories ${ }^{8-12}$ of the metalinsulator transition. The effects of the transition can thus be characterized by measuring $\chi$ for $n \leq n_{c}$ and $\sigma(0)$ for $n \geq n_{c}$.

The uncertainty in the absolute values of $n( \pm 5 \%$, from room-temperature resistivities calibrated to nuclear decay ${ }^{13}$ of radioactive donors) prevents a determination of $\zeta$ for $n_{c} / n-1 \leqslant 5 \%$. We have determined relative $n$ values more accurately by fitting $4 \pi \chi^{\prime \prime}(T)$ [i.e., the conductivity at our measuring frequency $\sigma(\omega, T)]$ to the form

$$
\sigma(\omega, T)=\sigma_{0}(\omega, 0)+\sigma_{1} \exp \left[-\left(T_{0} / T\right)^{1 / 4}\right] .
$$

The constant $\sigma_{0}(\omega, 0)$ increases monotonically as $n$ approaches $n_{c}$. The second term assumes the form of variable range hopping ${ }^{14}$ at the lowest temperatures with the characteristic hopping temperature $T_{0}$. Consistent with our results, we assume that $T_{0}$ scales linearly with $\left(n_{c} / n-1\right)$, so that we can eliminate $n_{c}$ as a parameter in determining $\zeta$. Equation (3) is fitted to the data over the lowest decade in temperature, except for the sample closest to $n_{c}$ whose high conductivity restricts the fit to $T<50 \mathrm{mK}$.

As shown in Fig. 3, we plot $(4 \pi \chi)^{-1 / 1.15}$ versus the fitted value of $T_{0}$. The good fit shown by the straight line indicates that $\chi \propto T_{0}^{-1.15 \pm 0.15}$ and so, assuming $T_{0} \propto\left(n_{c} / n-1\right)$, we have $\zeta=1.15 \pm 0.15$ in agreement with previous results ${ }^{2,15}$ at larger $n_{c} / n-1$. For the sample closest to the transition $\left(n_{c} / n-1 \leq 0.01\right)$, the small but finite measuring frequency probably makes $4 \pi \chi=720$ an underestimate. We have indicated the uncertainty involved in extrapolating to $\omega=0$ by the vertical error bar in Fig. 3. We believe the uncertainty in this extrapolation is insignificant except for the sample nearest to the critical density.

The scaling theory of localization ${ }^{9-12}$ predicts $\sigma(0) \propto 1 / \xi$, where the scale length near $n_{c}$ has the critical form $\xi=\xi_{0}\left(n / n_{c}-1\right)^{-\nu}$. McMillan, ${ }^{10}$ using scaling arguments for quantum diffusion, predicts $\chi \propto \zeta^{(\eta-1)}$, and thus $\zeta=\nu(\eta-1)$, where $1<\eta<3$. Other arguments ${ }^{5,11,12}$ suggest that $\zeta=2 \nu$. If we combine our value of $\zeta$ with results for $\sigma(0)$ at $n>n_{c}$, where $\nu=0.48 \pm 0.07$, we have $\zeta / \nu$ $=2.3 \pm 0.5$.

Within McMillan's formulation, we obtain $\eta=3.3 \pm 0.5$, implying that the system is near the limit of no Coulomb interactions where $\eta=3$. However, a variety of measurements ${ }^{16,17}$ in similar sys- 


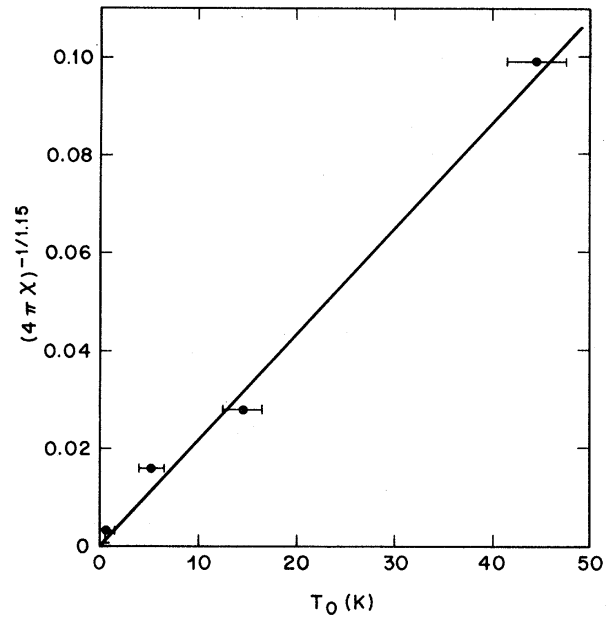

FIG. 3. Real part of the donor dielectric susceptibility $4 \pi X$ as a function of $T_{0}$ from Eq. (3). The straight line through the data with $(4 \pi \chi)^{-1 / 1.15}$ plotted, combined with the assumption that $T_{0} \propto n_{c} / n-1$, implies that $4 \pi \chi=\chi_{0}\left(n_{c} / n-1\right)^{\zeta}$ with $\zeta=1.15 \pm 0.15$. This behavior agrees with previous results as shown in Fig. 2, but is unexplained theoretically. tems suggests that these interactions are strong. A further difficulty is that McMillan ${ }^{10}$ has interpreted the tunneling results ${ }^{16}$ in metallic granular $\mathrm{Al}$ as indicating $\eta \simeq 2$. These inconsistencies suggest that further theoretical analysis is needed. Alternatively, if we assume $\zeta=2 \nu$, then we can solve for $\nu$ $=0.58 \pm 0.08$ from our $\chi$ data. This value is consistent with that from the $\sigma(0)$ measurements, but both values of $\nu$ disagree with the estimates ${ }^{9,10}$ from scaling theories of localization which give $\nu \sim 1$.

In summary, we have measured the zerotemperature donor dielectric susceptibility at densities closer to the metal-insulator transition than those of previous studies in doped semiconductors. We find that the critical divergence of $4 \pi \chi$ agrees with previous experimental results, but it is not explained by existing scaling theories of the transition.

\section{ACKNOWLEDGMENT}

The work at Princeton University was partially supported by Grant No. DMR 81-04414 from the NSF.
"Also at Joseph Henry Laboratory, Princeton University, Princeton, N.J. 08544.

${ }^{1}$ N. O. Lipari and D. L. Dexter, Phys. Rev. B $\underline{18}, 1346$ (1978).

${ }^{2}$ M. Capizzi, G. A. Thomas, F. DeRosa, R. N. Bhatt, and T. M. Rice, Phys. Rev. Lett. 44, 1019 (1980).

${ }^{3}$ P. Townsend, J. Phys. C 11,1481 (1978), has obtained $\chi$ by analyzing the oscillations due to interference in the transmitted intensity as a function of far-infrared frequency.

${ }^{4}$ T. G. Castner, N. K. Lee, G. S. Cieloszyk, and G. L. Salinger, Phys. Rev. Lett. 34, 1627 (1975); J. Bethin, T. G. Castner, and N. K. Lee, Solid State Commun. 14, 1321 (1974). Preliminary evidence has been obtained indicating very large values of $4 \pi \chi$ in Si:As, T. G. Castner (private communication)

${ }^{5}$ T. F. Rosenbaum, K. Andres, G. A. Thomas, and R. N. Bhatt, Phys. Rev. Lett. 45, 1723 (1980).

${ }^{6} \mathrm{G}$. A. Thomas, Y. Ootuka, S. Kobayashi, and W. Sasaki, Phys. Rev. B 24, 4886 (1981).

${ }^{7}$ M. Paalanen, T. F. Rosenbaum, G. A. Thomas, and R. N. Bhatt (unpublished).

8P. W. Anderson, Phys. Rev. 109, 1492 (1958); D. J. Thouless, Phys. Rev. C 13, 93 (1974); F. J. Wegner, Z. Phys. B 25, 327 (1976).

${ }^{9}$ E. Abrahams, P. W. Anderson, D. C. Licciardello, and T.
V. Ramakrishnan, Phys. Rev. Lett. 42, 673 (1979); Y. Imry, ibid. 44, 469 (1980).

${ }^{10}$ W. L. McMillan, Phys. Rev. B 24, 2739 (1981).

${ }^{11}$ P. Belitz, A. Gold, and W. Götze, Z. Phys. B 44, 273 (1981), and references therein.

${ }^{12}$ Y. Imry, Y. Gefen, and D. J. Bergman, in Proceedings Taniguchi Symposium, edited by Y. Nagaoka (Springer, New York, 1982) (in press).

${ }^{13}$ F. Mousty, P. Ostoja, and L. Passari, J. Appl. Phys. $\underline{45}$, 4576 (1974).

${ }^{14}$ N. F. Mott and E. A. Davis, Electronic Processes in NonCrystalline Materials (Oxford University Press, Oxford, 1979), p. 32ff.

${ }^{15} \mathrm{An}$ analysis of $4 \pi \chi$ measurements as a function of $n$ in Si:As yields a value of $\zeta$ consistent with our results; T. G. Castner (private communication).

${ }^{16}$ R. C. Dynes and J. P. Garno, Phys. Rev. Lett. 46, 137 (1981).

${ }^{17}$ T. F. Rosenbaum, K. Andres, G. A. Thomas, and P. A. Lee, Phys. Rev. Lett. 46, 568 (1981); T. F. Rosenbaum, R. F. Milligan, G. A. Thomas, P. A. Lee, T. V. Ramakrishnan, R. N. Bhatt, K. DeConde, H. Hess, and T. Perry, ibid. 47, 1758 (1981); G. A. Thomas, Y. Ootuka, S. Katsumoto, S. Kobayashi, and W. Sasaki, Phys. Rev. B (in press). 